\title{
OPTIMIZATION OF PILOT POWER FOR SERVICE COVERAGE AND SMOOTH HANDOVER IN WCDMA NETWORKS
}

\author{
Iana Siomina and Di Yuan \\ Department of Science and Technology, Linköping University \\ SE-601 74 Norrköping, Sweden \\ iansi, diyua@itn.liu.se
}

\begin{abstract}
In WCDMA networks, the presence of a cell is announced through a common pilot channel (CPICH). The power levels of the pilot channels have a great impact on coverage and service quality. Using mathematical optimization techniques, we address the problem of minimizing the amount of pilot power for providing service coverage and smooth handover. We present numerical results for several realistic planning scenarios of WCDMA networks, and analyze the pilot power solutions found by optimization versus those obtained by ad hoc strategies.
\end{abstract}

Keywords: WCDMA, pilot power, coverage, handover, mathematical optimization.

\section{Introduction}

In a WCDMA network, a cell announces its presence through a common pilot channel (CPICH). Pilot signals provide channel estimation to mobile terminals, and thereby facilitate cell selection and handover. Mobile terminals continuously monitor pilot signals of the network. Typically, a mobile terminal is attached to the cell with the strongest pilot signal.

Pilot power levels strongly affect coverage and service [2]. The pilot power of a cell effectively determines the cell size, and, consequently, the traffic load in the cell. Thus, to optimize the network performance, the pilot power levels should be carefully chosen [12]. Previous work of analyzing the effect of pilot power on network performance can be found in, for example, [4, 6, 8-10, 13].

We study the problem of providing service in a WCDMA network using a minimum amount of pilot power. There are a couple of reasons for minimizing pilot power consumption. First, as the total power available to the network is limited, less amount of pilot power means more power for user traffic. (Typically, the pilot power of a cell lies somewhere between $5 \%$ and $10 \%$ of the 
cell power, [5].) A second reason for minimizing pilot power is to reduce pilot pollution and interference (e.g., $[1,6])$.

We consider two types of service constraints. The first constraint is full service coverage, that is, a mobile terminal must be able to receive at least one pilot signal anywhere in the network. This constraint is defined by imposing a carrier-to-interference ratio (CIR) target for the pilot signals. Second, the pilot signals are planned to enable smooth handover. Here, by smooth handover, we mean that, when a mobile terminal moves across cell boundaries and changes its home cell, the handover operation can be performed with minimum risk of dropping a call or disrupting a data session. To enable smooth handover, the CIR target is enforced not only in the interior of a cell, but also at its boundaries to other cells. Mathematically, these two types of constraints are of the same characteristic; they are hence formulated using one set of constraints in our mathematical formulations. In our numerical experiments, we study several realistic planning scenarios of WCDMA networks, including two city networks in Europe. We analyze the pilot power solutions found by our optimization technique versus those obtained using two ad hoc approaches. Our numerical study also provides some insights into the impact of the constraint of smooth handover on pilot power consumption.

The remainder of the paper is organized as follows. In Section 2 we describe our system model. The optimization problem is formalized in Section 3, and two ad hoc solutions are presented in Section 4. Mathematical formulations are discussed in Section 5, and a Lagrangean heuristic is described in Section 6. We present our numerical study in Section 7. Finally, in Section 8 we draw some conclusions and discuss forthcoming research.

\section{System Model}

\subsection{Preliminaries}

Consider a WCDMA network consisting of $m$ cells. Let $P_{i}^{T o t}$ denote the total transmission power available in cell $i$. This amount of power is shared among the pilot channel, other signaling channels, as well as user traffic. We use $x_{i}$ to denote the pilot power of cell $i$.

The service area is represented by a grid of bins, for which predictions (or measurements) of signal propagation are performed. Let $n$ denote the number of bins, and $g_{i j}$ the power gain between the antenna of cell $i$ and bin $j$. Thus, in bin $j$, the power of the received pilot signal of cell $i$ is $x_{i} g_{i j}$. The interference experienced by a mobile terminal in bin $j$, with respect to cell $i$, reads $I_{i j}=$ $\left(1-\alpha_{j}\right) P_{i} g_{i j}+\sum_{k \neq i} P_{k} g_{k j}+\nu_{j}$, where $P_{i}$ and $P_{k}$ are the tranmission power of cells $i$ and $k$, respectively. Parameter $\alpha_{j} \in(0,1)$ is the orthogonality factor in bin $j$, and $\nu_{j}$ represents the effect of the thermal noise in bin $j$. 
We consider planning scenarios with high traffic load, and assume therefore that all base stations operate at full power, i.e., $P_{i}=P_{i}^{\text {Tot }}, i=1, \ldots, m$. This corresponds to the worst-case interference scenario. We can then write $I_{i j}$ as

$$
I_{i j}=\left(1-\alpha_{j}\right) P_{i}^{T o t} g_{i j}+\sum_{k \neq i} P_{k}^{T o t} g_{k j}+\nu_{j}
$$

The strength of a pilot signal is defined by its CIR. For cell $i$ and bin $j$, the CIR is

$$
\gamma_{i j}=\frac{x_{i} g_{i j}}{I_{i j}}=\frac{x_{i} g_{i j}}{\left(1-\alpha_{j}\right) P_{i}^{T o t} g_{i j}+\sum_{k \neq i} P_{k}^{T o t} g_{k j}+\nu_{j}}
$$

\subsection{Service Constraints}

Two service constraints are taken into account in our planning problem. The first constraint is full coverage. A necessary condition for a mobile terminal to access any network service is the detection of at least one pilot signal. We assume that, to successfully detect a pilot signal, the CIR must meet a threshold $\gamma_{0}$. Thus, the service of cell $i$ is available in bin $j$ only if the following is true.

$$
\gamma_{i j} \geq \gamma_{0}
$$

Full service coverage means that for any bin, there are one or more cells, for which (3) holds. Pilot power minimization subject to (3) has been previously studied in [11].

The second service constraint involves smooth handover. Handover occurs when a mobile terminal moves from the service area of one cell to that of another. Full service coverage does not necessarily ensure smooth handover. For example, consider two adjacent bins served by two different cells, for which the CIR of each of the two pilot signals is good in its respective bin, but very poor in the other. A mobile terminal that moves from one bin into the other, crossing the boundary of its home cell, may have difficulties in detecting the pilot signal of the other cell in time. When this occurs, there is a risk of dropping a call or interrupting a data session.

To facilitate smooth handover, a mobile terminal should be able to detect the pilot signal of the cell to which handover will take place, before it leaves its current home cell. For this purpose, the pilot power levels should be set such that, for the above example, the pilot signal of a cell not only covers its own bin, but also provides some coverage in the adjacent bin served by the other cell. One way to provide this kind of coverage is to require that the CIR of a pilot signal is above $\gamma_{0}$ in bins adjacent to the current cell. However, 
this may lead to an unreasonably (and unnecessarily) large amount of pilot power, and a risk of pilot pollution. Instead, we handle smooth handover by requiring that, if two adjacent bins belong to different cells, both pilot signals have a CIR of at least $\gamma_{0}$ at the boundary of the two bins. (This requirement increases the likelihood of being in soft or softer handover for mobile terminals at cell boundaries.) Modeling this constraint would require prediction of signal propagation at bin boundaries. Such predictions are not available in our system model. (An implicit assumption in Section 2.1 is that, for every bin, the power gain of a cell is identical in the entire bin.) However, it is reasonable to assume that, for two adjacent bins, the power gain at their boundary is somewhere between the gain values of the two bins. In this paper, we use the average value of the two power gain values to represent the power gain at the boundary.

Consider cell $i$ and two adjacent bins $j_{1}$ and $j_{2}$. If $x_{i}$ meets the CIR target in both bins, or in none of the two, the aforementioned constraint of smooth handover does not apply. Assume that cell $i$ has a sufficiently high pilot CIR in bin $j_{1}$ but not bin $j_{2}$, and that $g_{i j_{1}}>g_{i j_{2}}$ (because otherwise the CIR in $j_{2}$ is at least as good as that in $j_{1}$ ). To enable smooth handover for mobile terminals moving from $j_{2}$ into $j_{1}$, the strength of the received pilot signal is calculated using the average value of $g_{i j_{1}}$ and $g_{i j_{2}}$. The new CIR formula is as follows.

$$
\frac{x_{i} \frac{\left(g_{i j_{1}}+g_{i j_{2}}\right)}{2}}{I_{i j_{1}}}=\frac{x_{i} \frac{\left(g_{i j_{1}}+g_{i j_{2}}\right)}{2}}{\left(1-\alpha_{j_{1}}\right) P_{i}^{T o t} g_{i j_{1}}+\sum_{k \neq i} P_{k}^{T o t} g_{k j_{1}}+\nu_{j_{1}}} .
$$

Note that, the interference computation in (4) uses the power gain of bin $\dot{\lambda}$ (i.e., same as in (1)), not the average power gain. The reason for this is simple: The pilot power levels are planned for the scenario of worst-case interference - using the average power gain in the denominator of (4) would lead to less interference and thus jeopardize full coverage.

To formalize the constraint of smooth handover, we use $A(j)$ to denote the set of adjacent bins of bin $j$. For most bins, this set contains eight elements. If cell $i$ satisfies (3), then the new CIR formula applies for all bins in $A(j)$. For convenience, we introduce the notation $\bar{g}_{i j}$ to represent the new, adjusted power gain for bin $j$, that is, $\bar{g}_{i j}=\min \left\{g_{i j}, \min _{l \in A(j)} \frac{g_{i l}+g_{i j}}{2}\right\}$. We can then write the constraint of smooth handover as follows.

$$
\bar{\gamma}_{i j}=\frac{x_{i} \bar{g}_{i j}}{I_{i j}}=\frac{x_{i} \bar{g}_{i j}}{\left(1-\alpha_{j}\right) P_{i}^{T o t} g_{i j}+\sum_{k \neq i} P_{k}^{T o t} g_{k j}+\nu_{j}} \geq \gamma_{0} .
$$

Examining the two service constraints, (3) and (5), we observe that the latter is always as least as strong as the former. Therefore, if cell $i$ covers bin $j$, the pilot power must be at least $\bar{P}_{i j}$, which is derived from (5): 


$$
x_{i} \geq \bar{P}_{\imath j}=\frac{\gamma_{0}\left(\left(1-\alpha_{j}\right) P_{i}^{T o t} g_{i j}+\sum_{k \neq i} P_{k}^{T o t} g_{k j}+\nu_{j}\right)}{\bar{g}_{i j}} .
$$

Remark We impose (5) regardless of whether bin $j$ lies on the boundary of cell $i$ or not. Suppose that bin $j$ is in the interior of cell $i$, and, consequently, (3) is satisfied for bin $j$ as well as for all bins in $A(j)$. In this case, it can be easily realized that constraint (5) is also satisfied and thus redundant. As a result, the impact of (5) on the pilot power of a cell is determined by those bins on the cell boundary.

\section{Problem Definition}

Our pilot power optimization problem, which we denote by PPOP, is defined as follows.

- Objective: Minimize the total pilot power, i.e., $\min \sum_{i=1}^{m} x_{i}$.

- Constraint one: Every bin is covered by at least one pilot signal, that is, for any bin $j$, there exists at least one cell $i$ for which $x_{i} \geq \widetilde{P}_{i j}$.

- Constraint two: The pilot power of cell $i$ is limited by $P_{i}^{T o t}$, i.e., $x_{i} \leq$ $P_{i}^{T o t}, i=1, \ldots, m$.

The following proposition states the computational complexity of PPOP.

Proposition 1 PPOP is $\mathcal{N P}$-hard.

Proof See the first appendix at the end of the paper.

\section{Two Ad Hoc Solutions}

One ad hoc solution to PPOP is the one in which all cells use the same level of pilot power, which we refer to as the solution of uniform pilot power. We use $P^{U}$ to denote the minimum (total) power of uniform-power solutions that satisfy the constraints of PPOP. The value of $P^{U}$ can be derived quite easily. Let $P_{j}^{U}=\min _{i=1, \ldots, m} \bar{P}_{i j}$. To provide service in bin $j$, the power of at least one pilot signal must be greater than or equal to $P_{j}^{U}$. As this is true for any bin, taking the maximum of $P_{j}^{U}$ over the bins gives a lower bound on any feasible uniform power. In addition, setting all pilot power levels to $\max _{j=1, \ldots, n} P_{j}^{U}$ yields a feasible solution to PPOP. We have thus shown the following.

$$
P^{U}=m \cdot \max _{j=1, \ldots, n} P_{j}^{U}=m \cdot \max _{j=1, \ldots, n} \min _{i=1, \ldots, m} \bar{P}_{i j} .
$$

A second ad hoc approach to PPOP is a greedy heuristic. We call the solution generated by this heuristic the power-based pilot power, because for every 
bin, the heuristic chooses the cell for which the required power is minimal among all the cells. For bin $j$, we use $c(j)$ to denote the cell that minimizes $\bar{P}_{i j}$, that is, $c(j)=\arg \min _{i=1, \ldots, m} \bar{P}_{i j}$. The pilot power of cell $i$ is thus $P_{i}^{G}=\max _{j=1, \ldots, n: c(j)=i} \bar{P}_{i j}$. The total pilot power of this solution is therefore

$$
P^{G}=\sum_{i=1}^{m} P_{i}^{G}=\sum_{i=1}^{m} \max _{j=1, \ldots, n: c(j)=i} \bar{P}_{i j} .
$$

\section{Mathematical Formulations}

\subsection{A Cell-bin Formulation}

Problem PPOP can be formulated mathematically using the pilot power variables $x_{i}, i=1, \ldots, m$, and the following set of binary decision variables.

$$
y_{i j}= \begin{cases}1 & \text { if cell } i \text { covers bin } j, \text { i.e., } \bar{\gamma}_{i j} \geq \gamma_{0}, \text { or, equivalently, } x_{i} \geq \bar{P}_{i j}, \\ 0 & \text { otherwise. }\end{cases}
$$

Because the pilot power of a cell has an upper limit, not all cells are able to cover a bin. Therefore, we only need $y$-variables for feasible combinations of cells and bins. For this reason, we define a set $C(j)$, which consists of all cells that can cover bin $j$ using a feasible pilot power, i.e., $C(j)=\{i=1, \ldots, m$ : $\left.\bar{P}_{i j} \leq P_{i}^{T o t}\right\}$. PPOP can then be stated as follows.

$$
\begin{array}{ll}
\text { [PPOP-CB] } P^{*}=\min \sum_{i=1}^{m} x_{i} \\
\text { s.t. } & \sum_{i \in C(j)} y_{i j} \geq 1, \quad j=1, \ldots, n, \\
& \bar{P}_{i j} y_{i j} \leq x_{i}, \quad i \in C(j), j=1, \ldots, n, \\
& y_{i j} \in\{0,1\}, i \in C(j), j=1, \ldots, n .
\end{array}
$$

Constraints (10) ensure that every bin is covered by at least one cell. By (11), $x_{i}$ must be at least $\bar{P}_{i j}$, if cell $i$ covers bin $j$. The non-negativity restrictions on the $x$-variables are implicitly handled by (11).

\subsection{A Refined Formulation}

From a computational standpoint, formulation PPOP-CB is not efficient. In particular, its linear programming (LP) relaxation is very weak. Solving PPOP using this formulation is out of reach of a standard problem solver ${ }^{1}$. To avoid this weakness, we derive a second, refined formulation. The refinement is

\footnotetext{
${ }^{1}$ In our numerical study, CPLEX [3] did not manage to find optimal or near-optimal solutions within any reasonable amount of time even for the smallest test network ( 60 cells and 1375 bins).
} 
based on the rather simple observation that, in an optimal solution to PPOP, the pilot power of cell $i$ attains a value belonging to the discrete set $\left\{\vec{P}_{i j}, j=\right.$ $1, \ldots, n: i \in C(j)\}$. In the refined formulation, we use the following set of binary variables.

$$
z_{i k}= \begin{cases}1 & \text { if the pilot power of cell } i \text { equals } \bar{P}_{i k}, \\ 0 & \text { otherwise. }\end{cases}
$$

In Section 5.1, we defined the sets $C(j), j=1, \ldots, n$, each of which contains the set of cells that can cover a bin. In the refined formulation, it is more convenient to use the notation $B(i)$, which describes the possibility of coverage from the perspective of cells. Specifically, we let $B(i)=\left\{j: \bar{P}_{i j} \leq P_{i}^{T o t}\right\}$. Also, we define a set of indication parameters for the refined formulation:

$$
a_{i j k}= \begin{cases}1 & \text { if bin } j \text { is covered by cell } i, \text { provided that } z_{i k}=1 \\ 0 & \text { otherwise }\end{cases}
$$

Below we present the refined formulation.

$$
\begin{array}{ll}
\text { [PPOP-RF] } P^{*}=\min \sum_{i=1}^{m} \sum_{k \in B(i)} \bar{P}_{i k} z_{i k} \\
\text { s. t. } \quad \sum_{k \in B(i)} z_{i k}=1, i=1, \ldots, m, \\
& \sum_{i \in C(j)} \sum_{k \in B(i)} a_{i j k} z_{i k} \geq 1, j=1, \ldots, n, \\
& z_{i k} \in\{0,1\}, i=1, \ldots, m, k \in B(i) .
\end{array}
$$

In PPOP-RF, (14) states that exactly one of the possible pilot power levels is selected for every cell. By (15), every bin is covered by at least one cell.

Although it may not be trivial, it can be shown that the LP relaxation of PPOP-RF is always at least as strong as that of PPOP-CB.

Proposition 2 The LP relaxation of PPOP-RF is at least as strong as that of PPOP-CB. In addition, there exist instances for which the former is strictly better than the latter.

Proof See the second appendix at the end of the paper.

\section{A Lagrangean Heuristic}

For large-scale networks, it is time-consuming to solve PPOP-RF exactly using a standard solver. We therefore developed a Lagrangean heuristic as an approximate solution method. Due to space limitation, we will not present the algorithm in its full detail. In brief, the Lagrangean heuristic comprises two components. The first component is a Lagrangean relaxation, in which constraints (15) are relaxed using Lagrangean multipliers $\lambda_{j}, j=1, \ldots, n$. The 
relaxation decomposes into one easily-solved subproblem per cell. For cell $i$, the subproblem is to minimize the function $\sum_{k \in B(i)}\left(\bar{P}_{i k}-\sum_{j=1}^{n} \lambda_{j} a_{i j k}\right) z_{i k}$, subject to the constraint $\sum_{k \in B(i)} z_{i k}=1$. The Lagrangean dual is then solved using subgradient optimization.

The second component is a primal heuristic, in which the solution of the relaxation, if infeasible, is modified to a feasible solution. The heuristic consists of two phases. The first phase involves covering bins that are not covered by any cell in the solution of the relaxation. Among these bins, the heuristic selects the bin for which the cardinality of the set $|C(j)|$ is minimal. To cover this bin, the cell that needs a minimum amount of incremental power is chosen. This is then repeated until all bins are covered. In the second phase, the heuristic attempts to reduce the total pilot power by examining bins covered by multiple cells. For each of such bins, the heuristic identifies whether any cell can reduce its pilot power if the bin is removed from the coverage area of the cell. The second phase terminates when no improvement of this type is identified.

The Lagrangean heuristic solves the relaxation and applies the primal heuristic for a predefined number of subgradient optimization iterations. At termination, the Lagrangean heuristic yields both an upper bound (the best feasible solution found) and a lower bound (the best value of the Lagrangean relaxation) to the optimum.

\section{Numerical Study}

We used three WCDMA networks in our numerical study. The first network was provided by Ericsson Research, Sweden. The other two networks, provided by the MOMENTUM project [7], originate from planning scenarios for Berlin and Lisbon, respectively. Table 1 displays some network characteristics.

Table 1. Network characteristics. ${ }^{a}$

\begin{tabular}{lrrr}
\hline & Network N1 & Network N2 & Network N3 \\
\hline Sites & 22 & 50 & 52 \\
Cells & 60 & 148 & 140 \\
Bins & 1375 & 22500 & 62500 \\
Bin size $\left(\mathrm{m}^{2}\right)$ & $40 \times 40$ & $50 \times 50$ & $20 \times 20$ \\
$P_{i}^{\text {Tot }}, i=1, \ldots, m$ & $20 \mathrm{~W}$ & $19.95 \mathrm{~W}$ & $19.95 \mathrm{~W}$ \\
$\gamma_{0}$ & $-18.24 \mathrm{~dB}$ & $-20 \mathrm{~dB}$ & $-20 \mathrm{~dB}$ \\
$\nu_{j}, j=1, \ldots, n$ & $-100 \mathrm{dbm}$ & $-108.1 \mathrm{dbm}$ & $-100 \mathrm{dbm}$ \\
$\alpha_{j}, j=1, \ldots, n$ & 0.4 & $\{0.327,0.633,0.938\}$ & $\{0.327,0.633,0.938\}$ \\
\hline
\end{tabular}

${ }^{a}$ For networks N1 and N3, the orthogonality parameter $\alpha_{j}$ depends on bin type (urban, rural, or mixed). 
For N1, we used a standard solver [3] to find the optimal solution using PPOP-RF. For N2 and N3, we applied the Lagrangean heuristic described in Section 6. We present our main results in Table 2, which displays the optimized pilot power, and the pilot power of the two ad hoc solutions.

Table 2. Pilot power solutions. ${ }^{a}$

\begin{tabular}{lrrrrrr}
\hline Network & \multicolumn{2}{c}{ Uniform pilot power } & \multicolumn{2}{c}{ Power-based pilot power } & \multicolumn{2}{c}{ Optimized pilot power } \\
& Total & Average & Total & Average & Total & Average \\
N1 & 92.58 & 1.54 & 46.87 & 0.78 & 41.52 & 0.69 \\
N2 & 464.40 & 3.13 & 192.51 & 1.30 & 160.00 & 1.08 \\
N3 & 415.28 & 2.97 & 174.20 & 1.24 & 147.83 & 1.06 \\
\hline
\end{tabular}

${ }^{a}$ For the optimized pilot power, its worst-case deviation from optimum, in a relative sense, are $9 \%$ and $12 \%$ for $\mathrm{N} 2$ and $\mathrm{N} 3$, respectively.

We observe that the power-based solution offers a substantial improvement over the solution of uniform pilot power. The former is, however, still quite far away from optimum, when compared to optimized pilot power, which corresponds to only a few percent of the total power available. Our results suggest, therefore, that it is possible to use a small amount of pilot power for providing service coverage and smooth handover in WCDMA networks.

The optimized pilot power levels of network N1 are further examined using a histogram in Figure 1. We conclude that the power levels of most pilot signals lie between $0.3 \mathrm{~W}$ and $1.0 \mathrm{~W}$, and, in addition, most cells use a pilot power that is less than the average $(0.69 \mathrm{~W}$ in this case).

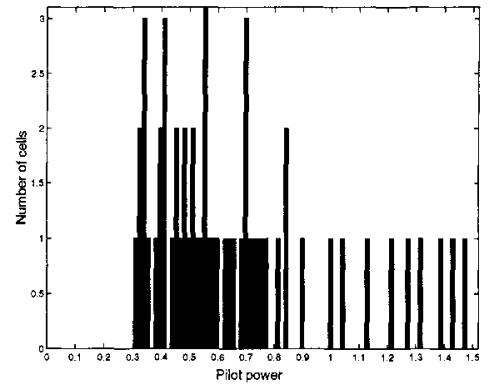

Figure 1. A histogram of the pilot power of network N1.

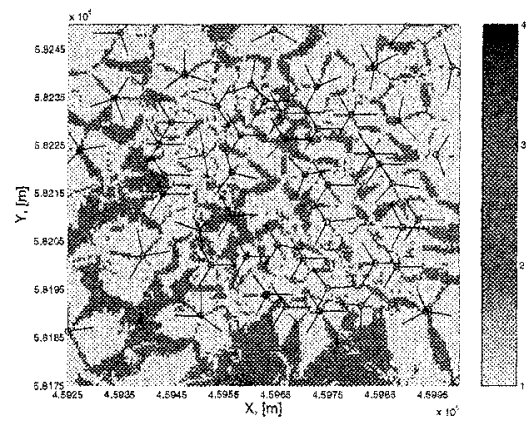

Figure 2. Pilot signal coverage of network N2 (city of Berlin).

Figure 2 illustrates the pilot signal coverage of network N2 (city of Berlin). For each bin, its color (or darkness) represents the number of cells providing coverage in the bin. The figure also shows the locations of the base stations as 
well as the antenna directions. We observe that most parts of the service area are covered by one or two pilot signals. (A more detailed examination of the coverage statistics shows that about $32 \%$ of the bins are covered by more than one pilot signal.) In many parts of the figure, bins covered by multiple cells form lines that indicate cell boundaries.

In the next part of our numerical study, we examine the impact of the constraint of smooth handover on pilot power. Ignoring smooth handover, the pilot signals need to satisfy constraint (3) only, i.e., if cell $i$ covers bin $j$, the minimum pilot power is $P_{i j}$, which is derived from (3): $P_{i j}=\gamma_{0} \cdot((1-$ $\left.\left.\alpha_{j}\right) P_{i}^{T o t} g_{i j}+\sum_{k \neq i} P_{k}^{T o t} g_{k j}+\nu_{j}\right) / g_{i j}$. We replace $\bar{P}_{i j}$ by $P_{i j}$ in PPOP-RF, which then minimizes the total pilot power without the constraint of smooth handover. The results are presented in Table 3. Comparing the results in this table to those in Table 2, we observe that between 30\% and 50\% additional pilot power are necessary to support smooth handover.

Table 3. Pilot power solutions without smooth handover. ${ }^{a}$

\begin{tabular}{lrrrrrr}
\hline Network & \multicolumn{2}{c}{ Uniform pilot power } & \multicolumn{2}{c}{ Power-based pilot power } & \multicolumn{2}{c}{ Optimized pilot power } \\
& Total & Average & Total & Average & Total & Average \\
N1 & 64.24 & 1.09 & 31.61 & 0.53 & 27.87 & 0.46 \\
N2 & 356.44 & 2.41 & 146.56 & 0.99 & 114.80 & 0.78 \\
N3 & 276.79 & 1.98 & 133.64 & 0.95 & 110.14 & 0.79 \\
\hline
\end{tabular}

${ }^{a}$ For the optimized pilot power, its worst-case deviation from optimum, in a relative sense, are $6 \%$ and $12 \%$ for $\mathrm{N} 2$ and $\mathrm{N} 3$, respectively.

\section{Conclusions}

We have studied the problem of minimizing pilot power of WCDMA networks subject to service coverage and smooth handover. Several conclusions can be drawn from our study. First, both full coverage and smooth handover can be achieved using only a few percent of the total power in a network, even for the scenario of worst-case interference. Second, ensuring smooth handover in addition to full coverage results in a moderate increase in pilot power (less than 50\%). Moreover, our study shows that optimized pilot power considerably outperforms ad hoc approaches, and, therefore, mathematical models can be very helpful for optimizing power efficiency in WCDMA networks.

An extension of the current research is pilot power optimization for the purpose of load balancing. Because pilot signals influence cell size, pilot power can be adjusted to equalize the load over cells. This topic is to be addressed in forthcoming research. 


\section{Acknowledgments}

The authors wish to thank the research group at Ericsson Research, Linköping, Sweden, for the technical discussions and the data of the first test network. We also thank the group of the MOMENTUM project [7] for providing us with the test networks of Berlin and Lisbon. This work is partially financed by CENIIT (Center for Industrial Information Technology), Linköping Institute of Technology, Sweden.

\section{Appendix: Proof of Proposition 1}

We show that any instance of the minimum-cost set covering problem (which is $\mathcal{N P}$-hard) can be polynomially transformed to an instance of PPOP. Consider an instance of the set covering problem, where $\left\{S_{1}, S_{2}, \ldots, S_{m}\right\}$ is a collection of sets, and $B$ is a set of items. A set $S_{i}$ is associated with a cost $c_{i}$, and contains some (possibly all) of the items in $B$. The objective of the set covering problem is to select a subset of $\left\{S_{1}, S_{2}, \ldots, S_{m}\right\}$ at minimum cost, such that all the items in $B$ are included. The corresponding instance of PPOP has $m$ cells and $m+|B|$ bins. We choose the parameters $\bar{P}_{i j}$ and $P_{i}^{T o t}$ as follows.

- For bin $j=1, \ldots, m, \bar{P}_{j j}=\epsilon$, where $\epsilon$ satisfies $0<\epsilon<\min _{i=1, \ldots, m} c_{i}$, and $\bar{P}_{i j}>P_{i}^{T o t}, \forall i \neq j$. (That is, cell $j$ is the only cell that can cover bin $j$.)

- For cell $i=1, \ldots, m$ and $j=m+1, \ldots, m+|B|, \bar{P}_{i j}=c_{i}$ if $S_{i}$ contains the $(j-m)$ th item of set $B$, otherwise $\bar{P}_{i j}>P_{i}^{T o t}$.

The above transformation is clearly polynomial. Moreover, a feasible solution to the PPOP instance is also feasible to the set covering instance, and vice versa. Finally, for any such pair of solutions, the two objective functions have the same value. Hence the conclusion.

\section{Appendix: Proof of Proposition 2}

We prove the first part of the proposition by showing that, for any feasible solution to the LP relaxation of PPOP-RF, there is a corresponding solution to the LP relaxation of PPOP-CB, and, in addition, the total pilot power of the former is greater than or equal to the latter.

Consider a feasible solution, denoted by $\bar{z}=\left\{\bar{z}_{i k}, i=1, \ldots, m, k \in B(i)\right\}$, to the LP relaxation of PPOP-RF. Consider solution $\bar{y}=\left\{\bar{y}_{i j}, i \in C(j), j=1, \ldots, n\right\}$, where $\bar{y}_{i j}=\sum_{k \in B(i)} a_{i j k} \bar{z}_{i k}$, to the LP relaxation of PPOP-CB. It is easy to verify that $\bar{y}$ satisfies (10). Next, we show that the total power of $\bar{y}$ is at most as that of $\bar{z}$. For $\bar{y}$, the optimal value of $x_{i}$ in PPOP-CB is obviously $\bar{x}_{i}=\max _{j=1, \ldots, n: i \in C(j)} \bar{P}_{i j} \bar{y}_{i j}$. According to the definitions of the sets $B(i)$ and $C(j)$, this equation can also be written as $\bar{x}_{i}=\max _{j \in B(i)} \bar{P}_{i j} \bar{y}_{i j}$. Assume that the maximum occurs for bin $j^{*}$, i.e., $\bar{x}_{i}=\bar{P}_{i j^{*}} \bar{y}_{i j^{*}}$. For PPOP-RF, the pilot power of cell $i$ reads $\sum_{k \in B(i)} \bar{P}_{i k} \bar{z}_{i k}$. For cell $i$, let $\bar{B}(i)=\left\{k \in B(i): a_{i j^{*} k}=1\right\}$. The set $\bar{B}(i)$ contains all bins in $B(i)$ that, in order to be covered by cell $i$, require a pilot power of at least $\bar{P}_{i j^{*}}$. Then, $\sum_{k \in B(i)} \bar{P}_{i k} \bar{z}_{i k} \geq \sum_{k \in \bar{B}(i)} \bar{P}_{i k} \bar{z}_{i k} \geq \sum_{k \in \bar{B}(i)} \bar{P}_{i j^{*}} \bar{z}_{i k}=\bar{P}_{i j^{*}} \sum_{k \in \bar{B}(i)} \bar{z}_{i k}=$ $\bar{P}_{i j^{*}} \sum_{k \in B(i)} a_{i j^{*} k} \bar{z}_{i k}=\bar{P}_{i j^{*}} \bar{y}_{i j^{*}}=\bar{x}_{i}$. Because this holds for any cell, we have proved the first part of the proposition.

To show the second part of the proposition, it is sufficient to give an example. Consider two cells and four bins, where $\bar{P}_{11}=1.2, \bar{P}_{12}=0.8, \bar{P}_{13}=0.6, \bar{P}_{21}=0.6, \bar{P}_{22}=0.8$, and $\bar{P}_{24}=$ 0.3 . Assume also that $\bar{P}_{14}$ and $\bar{P}_{23}$ exceed their limits (and are thus irrelevant to the discussion). In the integer optimum, $y_{11}=y_{12}=y_{13}=y_{24}=1$, and the total pilot power equals 1.5 . The optimal LP solution of PPOP-CB is $y_{11}=0.5, y_{12}=0.75, y_{13}=1, y_{21}=0.6, y_{22}=0.25$, 
$y_{23}=1$, with a total power of 0.9 . (The relative gap is therefore $40 \%$.) The LP relaxation of PPOP-RF, on the other hand, yields the integer optimum.

\section{References}

[1] M. M. El-Said, A. Kumar, and A. S. Elmaghraby, Sensory system for early detection of pilot pollution interference in UMTS networks, in: Proceedings of the 10th International Conference on Telecommunications (ICT '03), Tahiti, Papeete, French Polynesia, February 2003, pp. 1323-1328,

[2] A. Eisenblätter, T. Koch, A. Martin, T. Achterberg, A. Fügenschuh, A. Koster, O. Wegel, and R. Wessäly, Modelling feasible network configurations for UMTS, in: Telecommunications Network Design and Management, eds. G. Anandalingam and S. Raghavan (Kluwer Academic Publishers, 2002) pp. 1-24.

[3] ILOG CPLEX 7.0, User's Manual (ILOG, August 2000).

[4] D. Kim, Y. Chang, and J. W. Lee, Pilot power control and service coverage support in CDMA mobile systems, Proceedings of IEEE VTC '99, Houston, TX, May 1999, pp. 1464-1468.

[5] J. Laiho, A. Wacker, and T. Novasad (eds.), Radio Network Planning and Optimisation for UMTS, (John Wiley \& Sons, 2002).

[6] R. T. Love, K. A. Beshir, D. Schaeffer, and R. S. Nikides, A pilot optimization technique for CDMA cellular systems, Proceedings of IEEE VTC '99, Houston, TX, May 1999, pp. $2238-2242$.

[7] MOMENTUM, http://momentum.zib.de (2003).

[8] Y. Sun, F. Gunnarsson, and K. Hiltunen, CPICH power settings in irregular WCDMA macro cellular networks, Proceedings of IEEE PIMRC '03, Beijing, China, September 2003, pp. 1176-1180.

[9] K. Valkealahti, A. Höglund, J. Pakkinen, and A. Flanagan, WCDMA common pilot power control with cost function minimization, Proceedings of IEEE VTC Fall '02, Vancouver, Canada, September 2002, pp. 2244-2247.

[10] K. Valkealahti, A. Höglund, J. Pakkinen, and A. Flanagan, WCDMA common pilot power control for load and coverage balancing, Proceedings of IEEE PIMRC'O2, Lisbon, Portugal, September 2002, pp. 1412-1416.

[11] P. Värbrand and D. Yuan, A mathematical programming approach for pilot power optimization in WCDMA networks, Proceedings of Australian Telecommunications Networks and Applications Conference (ATNAC), Melbourne, Australia, December 2003.

[12] J. Yang and J. Lin, Optimization of pilot power management in a CDMA radio network, Proceedings of IEEE VTC Fall 'O0, Boston, MA, September 2000, pp. 2642-2647.

[13] H. Zhu, T. Buot, R. Nagaike, and S. Harman, Load balancing in WCDMA systems by adjusting pilot power, Proceedings of the 5th International Symposium on Wireless Personal Multimedia Communications, Honolulu, Hawaii, October 2002, pp. 936-940. 